\title{
Stabilization of magnetic curvature-driven Rayleigh-Taylor instabilities
}

\author{
O G Onishchenko, O A Pokhotelov, Lennart Stenflo and P K Shukla
}

\section{Linköping University Post Print}

N.B.: When citing this work, cite the original article.

Original Publication:

O G Onishchenko, O A Pokhotelov, Lennart Stenflo and P K Shukla, Stabilization of magnetic curvature-driven Rayleigh-Taylor instabilities, 2012, Journal of Plasma Physics, (78), 93-97.

http://dx.doi.org/10.1017/S0022377811000444

Copyright: Cambridge University Press (CUP)

http://www.cambridge.org/uk/

Postprint available at: Linköping University Electronic Press

http://urn.kb.se/resolve?urn=urn:nbn:se:liu:diva-75104 


\title{
Stabilization of magnetic curvature-driven Rayleigh-Taylor instabilities
}

\author{
O. G. ON IS H C H E N K O ${ }^{1,2}$, O. A. POK H OTE L O V 1 , L. S T E N F L O ${ }^{3}$ and P. K. SH U K L A \\ ${ }^{1}$ Institute of Physics of the Earth, 10 B. Gruzunskaya Street, 123995 Moscow, Russia \\ (pokh@ifz.ru) \\ ${ }^{2}$ Space Research Institute, 84/32 Profsojuznaya Street, 117997 Moscow, Russia \\ ${ }^{3}$ Department of Physics, Linköping University, SE-58183 Linköping, Sweden \\ ${ }^{4}$ Institut für Theoretische Physik IV, Fakultät für Physik und Astronomie, Ruhr-Universität Bochum, \\ D-44780 Bochum, Germany
}

(Received 7 September 2011; revised 29 September 2011; accepted 30 September 2011; first published online 1 November 2011)

\begin{abstract}
The finite ion Larmor radius (FLR) stabilization of the magnetic curvaturedriven Rayleigh-Taylor (MCD RT) instability in a low beta plasma with nonzero ion temperature gradient is investigated. Finite electron temperature effects and ion temperature perturbations are incorporated. A new set of nonlinear equations for flute waves with arbitrary wavelengths as compared with the ion Larmor radius in a plasma with curved magnetic field lines is derived. Particular attention is paid to the waves with spatial scales of the order of the ion Larmor radius. In the linear limit, a Fourier transform of these equations yields an improved dispersion relation for flute waves. The dependence of the MCD RT instability growth rate on the equilibrium plasma parameters and the wavelengths is studied. The condition for which the instability cannot be stabilized by the FLR effects is found.
\end{abstract}

\section{Introduction}

Charged particle drifts in an inhomogeneous plasma immersed in magnetic and gravitational fields are associated with charge separation and the existence of electrostatic flute waves. In the region where gravitational acceleration and plasma density gradient are oppositely directed, the magnetic Rayleigh-Taylor (RT) instability can appear and generate flute waves. This instability is similar to the classical RT instability of an inhomogeneous fluid in a gravitational field. About 50 years ago it was shown [1-3] that the finite Larmor radius (FLR) effects have a stabilizing influence on this instability. However, the subsequent numerical simulations and detailed theoretical investigations $[4,5]$ showed that complete FLR stabilization is not always attainable. The previous investigations of the magnetic RT instability and nonlinear structures of flute waves were restricted to the long wavelength limit when the wave spatial scale is larger than the ion Larmor radius. Therefore, the conclusions $[4,5]$ on the possible absence of complete FLR stabilization in the wavelength region where the equations are not applicable, remained doubtful. Recently, in order to overcome this difficulty, the theory of flute waves, driven by the RT instability, was extended to the case of arbitrary spatial scales $[6,7]$. The analysis was carried out in the framework of both kinetic and hydrodynamic models. Particular attention was paid to flute waves with spatial scales of the order of the ion Larmor radius. It was found that complete FLR stabilization is absent when $g L_{N} / v_{T i}^{2}=1$, where $g$ is the gravitational acceleration, $L_{N}$ is the characteristic spatial scale of plasma inhomogeneity, and $v_{T i}$ is the ion thermal velocity.

The RT instability is one of the most important instabilities in space and laboratory plasmas where the role of the gravity force is often played by the magnetic field line curvature [8]. The present instability is analogous to the RT instability and is termed magnetic curvaturedriven (MCD) RT instability. The FLR stabilization of this instability has been studied in [9-13] in the long wavelength approximation. An extension of this analysis to the case of arbitrary wavelengths has been recently suggested in [14] with simplifying assumptions of cold electrons and isothermal equilibrium ion distributions. We note that in the analysis of RT instability in the curved magnetic field, the centrifugal acceleration of particles moving along the magnetic field lines was, for simplicity, replaced by effective acceleration due to gravity, i.e., by introducing $g_{\text {eff }}=v_{T i}^{2} / R$. Here $R$ is the local effective radius of the magnetic field line curvature. However, such an approach has the deficiencies of $[6,7]$ and [14] because the $\mathbf{E} \times \mathbf{B}$ drift in the curved magnetic field is not divergence-free. Thus, a rigorous consideration of MCD RT instability demands incorporation of the real magnetic field geometry.

An investigation of FLR stabilization of the MCD $\mathrm{RT}$ instability and related flute waves in a low- $\beta$ plasma with arbitrary spatial scales is the main purpose of the present study. In contrast to the previous analyses the effects of nonuniform ion temperature and nonzero electron temperature will also be incorporated. A model 
hydrodynamic approach that allows us to describe flute waves with spatial scales of the order of the ion Larmor radius in a plasma with finite ratio $R / L_{N}$ will be used. It will be shown that the electron temperature effects substantially modify the instability growth rate and the equilibrium condition at which the instability is not stabilized by the FLR effect. In this way we will thus significantly improve the results of $[6,7]$ and [14].

The paper is organized as follows: A closed set of equations for nonlinear flute waves with arbitrary spatial scales is deduced in Sec. 2. Section 3 describes the growth rate and the FLR stabilization of the MCD RT instability. Our discussion and conclusions are found in Sec. 4.

\section{Hydrodynamic equations}

We consider a weakly inhomogeneous low- $\beta$ plasma immersed in an external magnetic field $\mathbf{B}=B_{0} \hat{\mathbf{b}}, \hat{\mathbf{b}}=[(1-$ $x / R) \hat{\mathbf{z}}-(z / R) \hat{\mathbf{x}}]$, where $\hat{\mathbf{x}}$ and $\hat{\mathbf{z}}$ are unit vectors along the $x$ and $z$-axes in a local Cartesian coordinate system $(x, y, z)$. Here the $x$-axis is in the opposite direction to the plasma inhomogeneity gradient. We limit our consideration to low frequencies, $\omega_{c i}^{-1} d / d t \ll 1$, where $\omega_{c i}=$ $Z e B_{0} / m_{i}$ is the ion cyclotron frequency, $d / d t$ is the Lagrangian time derivative, $e$ and $m_{i}$ are the electron charge and ion mass, respectively, and $Z$ is the ion charge number. The inhomogeneous local unperturbed ion and electron densities, $n_{0 i}$ and $n_{0 e}$, are characterized by the spatial scale $L_{N}=\kappa_{N}^{-1}$, where $d \ln n_{0 i} / d x=d \ln n_{0 e} / d x=$ $-\kappa_{N}$. The unperturbed ion temperature is characterized by the spatial scale $L_{T}=\kappa_{T}^{-1}$, where $d \ln T_{0 i} / d x=$ $-\kappa_{T}$. We suppose that electrons are isothermal and the density/temperature gradients are negative, i.e., $\kappa_{N}>0$ and $\kappa_{T}>0$.

In order to describe ions in flute waves with arbitrary spatial scales as compared to the ion Larmor radius in a plasma with finite ion temperature gradient, we use the model hydrodynamic description [15]. The ion velocity is thus decomposed as

$$
\mathbf{v}_{i} \simeq \mathbf{v}_{E}+\mathbf{v}_{i D}+\mathbf{v}_{E}^{P}+\mathbf{v}_{i D}^{P}
$$

where $\mathbf{v}_{i D}=\left(1 / Z e B^{2}\right)\left(\mathbf{B} \times \nabla p_{i}\right)$ is the ion diamagnetic drift velocity, $p_{i}$ is the ion pressure, and $\mathbf{v}_{E}^{P}$ and $\mathbf{v}_{i D}^{P}$ stand for the polarization parts of the ion velocity. They are connected to $\mathbf{v}_{E}$ and $\mathbf{v}_{i D}$ through the relations

$$
\mathbf{v}_{E}^{P}=\frac{1}{\omega_{c i}}\left(\hat{\mathbf{z}} \times d_{t} \mathbf{v}_{E}\right) \text { and } \mathbf{v}_{i D}^{P}=\frac{1}{\omega_{c i}}\left(\hat{\mathbf{z}} \times d_{t} \mathbf{v}_{i D}\right) .
$$

Here as in [14-17] we denote $d_{t}$ as $d_{t} \equiv \partial / \partial t+\mathbf{v}_{E} \cdot \nabla$.

In order to describe the ion temperature perturbations, we use the ion thermal balance condition

$$
n_{i}\left(d_{t}+\mathbf{v}_{i D} \cdot \nabla\right) T_{i}+n_{i} T_{i} \nabla \cdot \mathbf{v}_{i}+\nabla \cdot \mathbf{q}_{\perp}=0,
$$

where $n_{i}$ and $T_{i}$ are the ion number density and temperature, respectively. The ion thermal flux $\mathbf{q}_{\perp}$ is given by

$$
\mathbf{q}_{\perp}=\frac{2 p_{i}}{m_{i} \omega_{c i}}\left(\hat{\mathbf{z}} \times \nabla_{\perp} T_{i}\right)-\frac{2}{m_{i} \omega_{c i}^{2}} d_{t}\left(p_{i} \nabla_{\perp} T_{i}\right)-\frac{2 v p_{i}}{m_{i} \omega_{c i}^{2}} \nabla_{\perp} T_{i},
$$

where $v$ is the ion-ion collision frequency, and $v \ll \omega_{c i}$.

Equations (3) and (4) correspond to two-dimensional ion motion for waves where the effective ratio of specific heats equals 2 . The first term on the right-hand side of (4) is the well-known ion thermal flux in Braginskii's hydrodynamics, where the numerical factor 2 instead of $5 / 2$ reflects the two-dimensional character of the ion motion. Furthermore, the second term corresponds to the so-called polarization part of the ion thermal flux in the Grad-type hydrodynamics. Finally, the third term describes the collisional thermal flux.

After substitution of the ion velocity (1) into the ion continuity equation, one obtains the dimensionless equation

$$
\begin{aligned}
& d_{\tau}\left(1-\nabla_{\perp}^{2}\right) \delta n-d_{\tau} \nabla_{\perp}^{2} \delta T+\left(\frac{1}{\hat{L}_{N}}-\frac{1}{\hat{R}}\right) \frac{\partial \Phi}{\partial Y}-d_{\tau} \nabla_{\perp}^{2} \Phi \\
& -\left\{\nabla_{\perp} \Phi, \nabla_{\perp} \delta p\right\}=-\hat{\mu} \nabla_{\perp}^{4} \Phi .
\end{aligned}
$$

Here $\delta n=\tilde{n}_{i} / n_{0 i}$, where $\tilde{n}_{i}=n_{i}-n_{0 i}$ is the perturbed ion number density, $\delta T=\left(T_{i}-T_{0 i}\right) / T_{0 i}$ is the normalized ion temperature perturbation, $\delta p=\tilde{p}_{i} / p_{i 0}$ is the normalized ion pressure perturbation, $\Phi=e \varphi / T_{i}$ is the normalized electrostatic potential, $\mathbf{E}_{\perp}=-\nabla_{\perp} \varphi$, the subscript $\perp$ denotes the vector component perpendicular to the ambient magnetic field, $\hat{L}_{N}=L_{N} / \rho_{i}$ and $\hat{R}=$ $R / 2 \rho_{i}$, where the numerical factor 2 has been introduced for notational convenience, $\rho_{i}=\left(T_{i} / m_{i}\right)^{1 / 2} / \omega_{c i}$ is the ion Larmor radius, $d_{\tau}=\partial / \partial \tau+\{\Phi, \ldots\}-(1 / \hat{R}) \partial / \partial Y$, $\{f, g\}=(\partial f / \partial X) \partial g / \partial Y-(\partial f / \partial Y) \partial g / \partial X$ is the Poisson bracket, and $\hat{\mu}=(3 / 10) v / \omega_{c i}$ is the dimensionless dynamic collision viscosity. In deriving (5) we used the fact that $\nabla \cdot \mathbf{v}_{E}=-2 E_{y} / B_{0} R$ and the relation $\nabla \cdot\left(\mathbf{v}_{i D} \tilde{n}_{i}\right)=\mathbf{v}_{i c} \cdot \nabla \tilde{n}_{i}$, where $\mathbf{v}_{i c}=-\left(2 v_{T i} \rho_{i} / R\right) \hat{\mathbf{y}}$ is the ion curvature drift velocity. We also normalized the space-time scales by $\rho_{i}$ and $\omega_{c i}^{-1}$, i.e., $\tau=t \omega_{c i}$ and $(X, Y)=(x, y) \rho_{i}^{-1}$.

The ion thermal balance condition in dimensionless form thus reduces to

$$
\begin{aligned}
& d_{\tau}\left(1-3 \nabla_{\perp}^{2}\right) \delta T-d_{\tau} \nabla_{\perp}^{2} \delta n-d_{\tau} \nabla_{\perp}^{2} \Phi+\left(\frac{1}{\hat{L}_{T}}-\frac{1}{\hat{R}}\right) \frac{\partial \Phi}{\partial Y} \\
& \quad+\left\{\nabla_{\perp} \Phi, \nabla_{\perp}(\delta p+2 \delta T)\right\}=\hat{v} \nabla_{\perp}^{2} \delta T,
\end{aligned}
$$

where $\hat{v}=2 v / \omega_{c i}$ is the normalized ion-ion collisional frequency.

Equations (5) and (6) must be supplemented by the electron continuity equation. In the low-frequency approximation we decompose the electron velocity as $\mathbf{v}_{e}=$ $\mathbf{v}_{E}+\mathbf{v}_{e D}$, where $\mathbf{v}_{e D}=-Z \tau_{i} \rho_{i}^{2} \omega_{c i}\left(B_{0} / B^{2}\right)\left(\mathbf{B} \times \nabla n_{e}\right)$ is the diamagnetic electron velocity and $\tau_{i}=T_{e} / T_{i}$ is the ratio of the electron to ion temperature. In dimensionless form the equation for the electron continuity is

$$
\frac{\partial \delta n_{e}}{\partial \tau}+\frac{Z \tau_{i}}{\hat{R}} \frac{\partial \delta n_{e}}{\partial Y}+\left(\frac{1}{\hat{L}_{N}}-\frac{1}{\hat{R}}\right) \frac{\partial \Phi}{\partial Y}=\left\{\delta n_{e}, \Phi\right\}+\hat{D} \nabla_{\perp}^{2} \delta n_{e} .
$$


Here $\delta n_{e}=\tilde{n}_{e} / n_{0 e}, \quad \tilde{n}_{e}=n_{e}-n_{0 e}$ is the perturbed electron number density, and $\hat{D}=\left(m_{e} / m_{i}\right) Z \tau_{i} \hat{v}_{e e}$ is the dimensionless diffusion coefficient. In the course of derivation of (7) we noted that $\nabla \cdot\left(n_{e} \mathbf{v}_{e D}\right)=\mathbf{v}_{e c} \cdot \nabla \tilde{n}_{e}$, where $\mathbf{v}_{e c}=\left(2 Z \tau_{i} v_{T i} \rho_{i} / R\right) \hat{\mathbf{y}}$ is the electron curvature drift velocity.

Equations (5)-(7) in the quasi-neutrality approximation, $\delta n=\delta n_{e}$, constitute a closed set of coupled equations for $\delta n, \Phi$, and $\delta T$ describing the nonlinear dynamics of MHD flute waves with arbitrary spatial scales. These equations are however quite cumbersome and can thus be used only for numerical simulations of the nonlinear dynamics of MCD RT instability. We note that in the ion isotropic approximation, $\delta T \rightarrow 0$, this set reduces to two equations i.e. (7) and

$$
\begin{aligned}
& d_{\tau}\left(1-\nabla_{\perp}^{2}\right) \delta n+\left(\frac{1}{\hat{L}_{N}}-\frac{1}{\hat{R}}\right) \frac{\partial \Phi}{\partial Y}-d_{\tau} \nabla_{\perp}^{2} \Phi \\
& -\left\{\nabla_{\perp} \Phi, \nabla_{\perp} \delta n\right\}=-\hat{\mu} \nabla_{\perp}^{4} \Phi .
\end{aligned}
$$

In the cold electron temperature limit, $\tau_{i}=T_{e} / T_{i} \rightarrow 0$, (7) and (8) coincide with the corresponding equations in [14]. Equations (7) and (8) in the large-scale approximation $\nabla_{\perp}^{2} \ll 1$ correspond to the equations analyzed in [9-13].

\section{Linear dispersion relations}

In the case of collisionless plasmas and in the linear approximation a Fourier transform of (7) gives the response of the normalized electron density perturbation to the perturbation of the electrostatic field,

$$
\delta n_{e}=-\frac{\omega_{N}-k_{y} v_{i c}}{\omega-k_{y} v_{e c}} \Phi,
$$

where $\omega_{N}=k_{y} v_{i D}$ is the ion drift frequency.

From (5) and (6) one finds a similar relation for the response of the normalized ion density perturbation to the electrostatic potential,

$\delta n=-\frac{\omega_{N}-k_{y} v_{i c}}{\omega-k_{y} v_{i c}} \Phi-\left(\alpha_{11} \frac{\omega-\omega_{N}}{\omega-k_{y} v_{i c}}-\alpha_{12} \frac{\omega_{T}-k_{y} v_{i c}}{\omega-k_{y} v_{i c}}\right) \Phi$,

where $\omega_{T}=\omega_{N} \eta, \eta=\kappa_{T} / \kappa_{N}, \alpha_{11}=z(1+2 z) /(1+4 z+$ $\left.2 z^{2}\right)$, and $\alpha_{12}=z /\left(1+4 z+2 z^{2}\right)$, where $z=k_{\perp}^{2} \rho_{i}^{2}$.

Using (9) and (10) in the charge electroneutrality approximation one obtains the dispersion relation

$$
\begin{aligned}
& \omega^{2}-\omega \omega_{N}\left[1-Z \tau_{i} \sigma+\frac{\alpha_{12}}{\alpha_{11}}(\eta-\sigma)\right] \\
& \quad+\omega_{c} \omega_{N}\left[\frac{1}{\alpha_{11}}\left(1+Z \tau_{i}\right)(1-\sigma)-Z \tau_{i}-Z \tau_{i} \frac{\alpha_{12}}{\alpha_{11}}(\eta-\sigma)\right] \\
& \quad=0
\end{aligned}
$$

and the normalized growth rate

$$
\begin{aligned}
\frac{\gamma}{\Gamma}= & \left\{\frac{z}{\alpha_{11}}\left(1+Z \tau_{i}\right)(1-\sigma)-Z \tau_{i} z-Z \tau_{i} z \frac{\alpha_{12}}{\alpha_{11}}(\eta-\sigma)\right. \\
& \left.-\frac{z}{4 \sigma}\left[1-Z \tau_{i} \sigma+\frac{\alpha_{12}}{\alpha_{11}}(\eta-\sigma)\right]^{2}\right\}^{1 / 2}
\end{aligned}
$$

Figures 1-3 illustrate the growth rate $\gamma / \Gamma$ as a function of $k_{\perp} \rho_{i}$ calculated with the help of (12) for different equilibrium parameters, $Z \tau_{i}=p_{e 0} / p_{i 0}, \sigma=2 L_{N} / R$, and $\eta \equiv L_{N} / L_{T}$. A comparison of the left and right panels shows that the instability growth rates depend weakly on the parameter $\eta$, when it varies from 0 to 0.5 . The critical value $z_{\text {cr }}$ at which the growth rate vanishes $\gamma\left(z=z_{\text {cr }}\right)=0$ depends strongly on the parameter $\hat{\sigma}=2\left(2+Z \tau_{i}\right) L_{N} / R$. The instability is stabilized by FLR effects when $\hat{\sigma}<1$ or $\hat{\sigma}>1$. For $\hat{\sigma}=1$, or $R=2\left(2+p_{e 0} / p_{i 0}\right) L_{N}$, the stabilization is absent, i.e., $z_{\mathrm{cr}} \rightarrow \infty$. In Fig. 1 the parameter $\hat{\sigma}$ is close to one and $z_{\mathrm{cr}} \gg 1$ at $Z \tau_{i}=10$. In Fig. $2 \hat{\sigma}=1.0$ at $Z \tau_{i}=3$, and in Fig. $3 \hat{\sigma}=0.9$ when $Z \tau_{i}=1$.

A rough estimate of plasma parameters for which the FLR stabilization of the RT instability is absent can be obtained by linearizing the simplified (7) and (8) that describe flute waves in the ion isothermal approximation. Using (8) one can find the response of the normalized ion density perturbation to the electrostatic potential,

$$
\delta n=-\frac{\omega_{N}-k_{y} v_{i c}}{\omega-k_{y} v_{i c}} \Phi-\alpha \frac{\omega-\omega_{N}}{\omega-k_{y} v_{i c}} \Phi
$$

where $\alpha=z /(1+z)$. With the help of (9) and (13) in the charged neutrality approximation, $\delta n_{e}=\delta n$, one thus obtains the dispersion relation

$$
\begin{aligned}
& \omega^{2}-\omega \omega_{N}\left(1-Z \tau_{i} \sigma\right)+\omega_{c} \omega_{N}\left[\frac{1}{\alpha}\left(1+Z \tau_{i}\right)(1-\sigma)-Z \tau_{i}\right] \\
& \quad=0
\end{aligned}
$$

where $\sigma=v_{i c} / v_{i D}=2 L_{N} / R, Z \tau_{i}=p_{e 0} / p_{i 0}$ and $\omega_{c}=$ $k_{y} v_{i c}$. From the dispersion relation (14) follows that the necessary condition for the instability is $\sigma<1$. The normalized instability growth rate is

$$
\frac{\gamma}{\Gamma}=\frac{|1-\hat{\sigma}|}{2 \sigma^{1 / 2}}\left(z_{\mathrm{cr}}-z\right)^{1 / 2},
$$

where

$$
z_{\text {cr }}=\frac{4 \sigma(1-\sigma)\left(1+Z \tau_{i}\right)}{(1-\hat{\sigma})^{2}} .
$$

The maximum growth rate is attained in the long wavelength limit, $k_{\perp} \rho_{i} \ll 1$. Moreover, the normalized growth rate is a factor $\left(1+Z \tau_{i}\right)^{1 / 2}$ larger than that for the cold electron limit [14]. If instead of the hydrodynamic expression $\alpha=z /(1+z)$ in (14) one inserts the kinetic theory expression [3] $\alpha^{K} \equiv 1-\exp (-z) I_{0}(z)$ then the critical kinetic value $z_{\mathrm{cr}}^{K}$ has to be found from

$$
\exp \left(-z_{\mathrm{cr}}^{K}\right) I_{0}\left(z_{\mathrm{cr}}^{K}\right)=\frac{(1-\hat{\sigma})^{2}}{\left(1+Z \tau_{i} \sigma\right)^{2}} .
$$



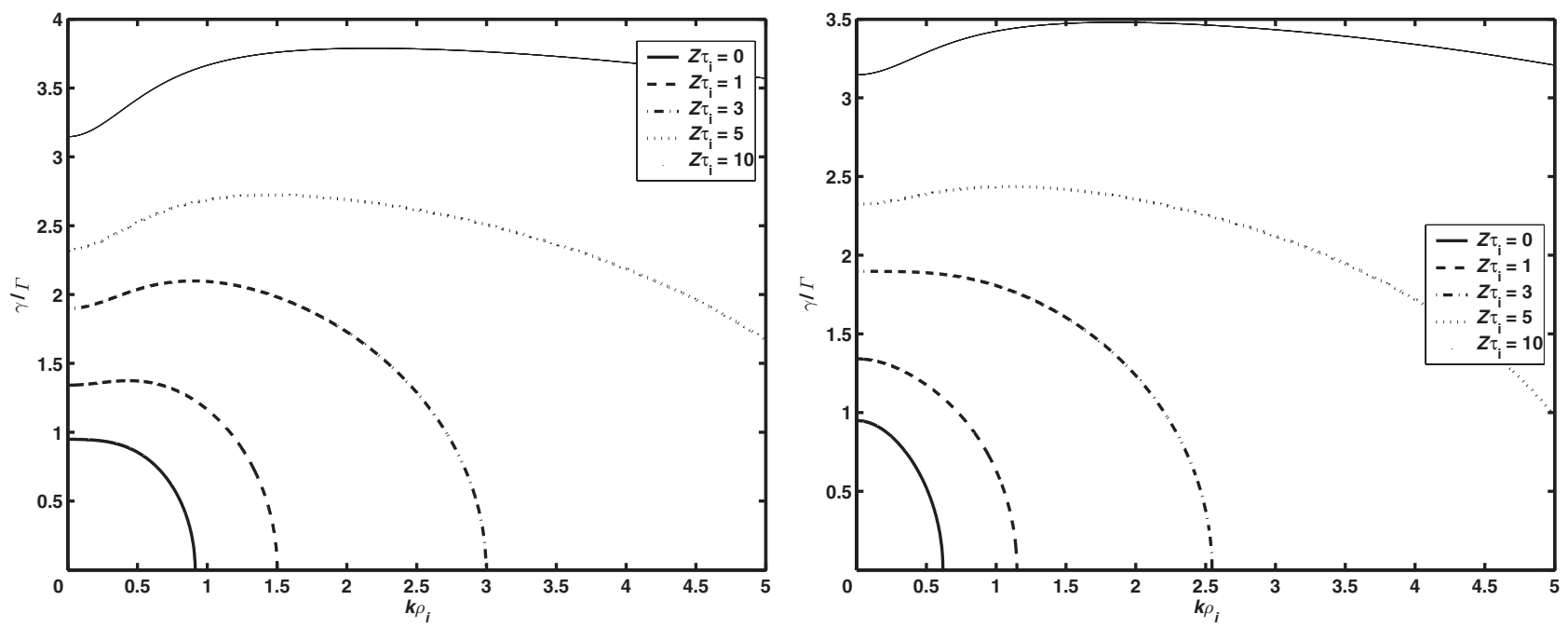

Figure 1. The normalized MCD RT instability growth rate as a function of $k_{\perp} \rho_{i}$ in plasmas with homogeneous ion temperature, $\eta=0$ and $\sigma=2 L_{N} / R=0.1$. The parameter $Z \tau_{i}=p_{e 0} / p_{i 0}$ takes the values $0,1,3,5$, and 10 (left panel). The normalized MCD RT instability growth rate as a function of $k_{\perp} \rho_{i}$ in plasmas with inhomogeneous ion temperature, $\eta=0.5$. The other parameters are the same as in Fig. 1, left panel.
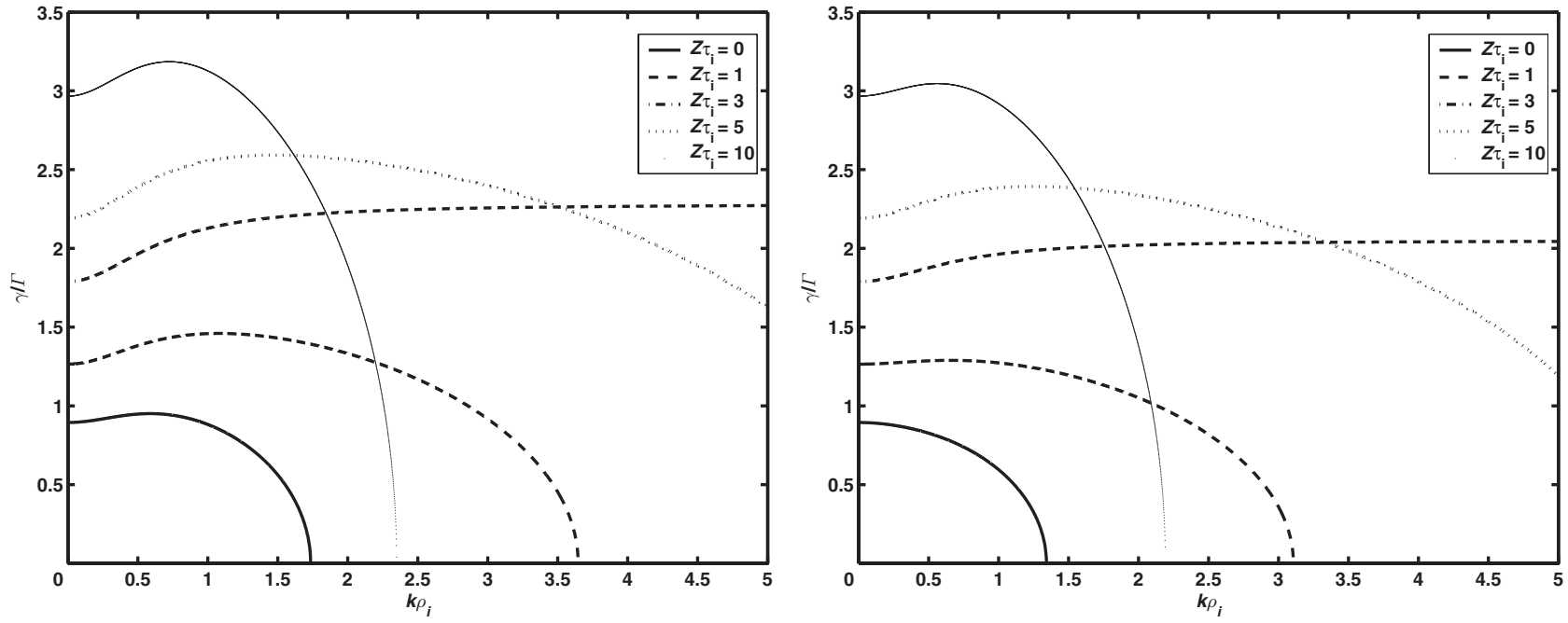

Figure 2. Same as in Fig. 1 but for $\sigma=0.2$.
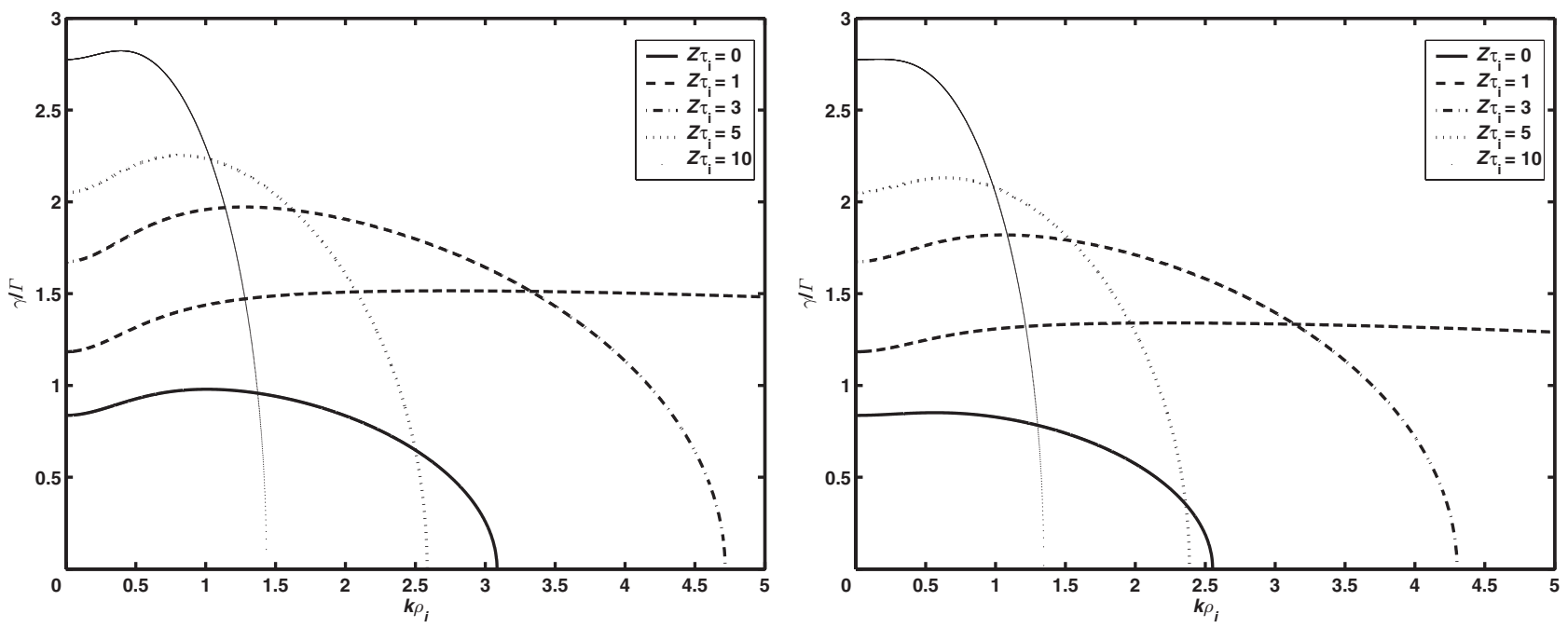

Figure 3. Same as in Fig. 1 but for $\sigma=0.3$. 
Equations (16) and (17) can be solved for the $z_{\mathrm{cr}}$ or $z_{\mathrm{cr}}^{K}$ except for the critical value $\hat{\sigma}=\sigma\left(2+Z \tau_{i}\right)=1$ or when $R / L_{N}=2\left(2+p_{e 0} / p_{i 0}\right)$.

Simple analytical estimates in the ion isothermal approximation, (15)-(17), show that the ion temperature perturbations weakly influence the instability stabilization. Figures 1-3 show that these perturbations in flute waves lead to a shift of the maximum growth rate from the long wavelength region $k_{\perp} \rho_{i} \ll 1$ to the region of short wavelengths of the order of the ion Larmor radius.

\section{Summary}

The present paper describes the FLR stabilization of the MCD RT instability and flute perturbations in a low- $\beta$ plasma when the spatial scales are arbitrary. It significantly extends our previous studies [6, 7, 14] of incomplete FLR stabilization to arbitrary ratios of the electron to ion pressure $Z \tau_{i}=p_{0 e} / p_{0 i}$. In addition, here the ion temperature perturbations are taken into account. New equations describing nonlinear flute waves with arbitrary spatial scales in a plasma with curved magnetic field lines have thus been derived. They are most relevant for numerical simulations of the nonlinear stage of instability. In the linear approximation a Fourier transform of these equations yields the dispersion relation for flute waves with arbitrary spatial scales in a curved magnetic field. The dependence of the instability growth rate on the equilibrium plasma parameters has been studied. It has been shown that when $R=2(2+$ $\left.p_{e 0} / p_{i 0}\right) L_{N}$, the FLR effects cannot stabilize the MCD RT instability.

\section{Acknowledgments}

This research was partially supported by the Russian Fund for Basic Research (grant nos. 10-05-00376 and
11-05-00920) and the Program of the Russian Academy of Sciences No 7.

\section{References}

[1] Rosenbluth, M. N., Krall, N. A. and Rostoker, N. 1962 Nucl. Fusion, Suppl. 1, 143.

[2] Roberts, K. V. and Taylor, J. B. 1962 Phys. Rev. Lett. 8, 197.

[3] Mikhailovskii, A. B. 1967 In: Reviews of Plasma Physics Vol. 3 (ed. M. A. Leontovich). New York: Plenum, 211 pp.

[4] Ferraro, N. M. and Jardin, S. C. 2006 Phys. Plasmas 13, 092101.

[5] Zhu, P., Schnack, D. D., Ebrahimi, F., Zweibel, E. G., Suzuki, M., Henga, C. C. and Sovinec, C. R. 2008 Phys. Rev. Lett. 101, 085005.

[6] Onishchenko, O. G. 2010 New frontiers in advanced plasma physics. AIP Conf. Proc. 1306, 145.

[7] Onishchenko, O. G., Pokhotelov, O. A., Stenflo, L. and Shukla, P. K. 2011 Phys. Plasmas 18, 022106.

[8] Kadomtsev, B. B. 1966 In: Reviews of Plasma Physics, Vol. 2 (ed. M. A. Leontovich). New York: Consultants Bureau, $153 \mathrm{pp}$.

[9] Das, A., Sen, A., Mahajan, S. and Kaw, P. 2001 Phys. Plasmas 8, 5104.

[10] Dastgeer, S., Singh, R., Nordman, H., Weiland, J. and Rogister, A. 2002 Phys. Rev. E 66, 036408.

[11] Sandberg, I. and Shukla, P. K. 2004 Phys. Plasmas 11, 542.

[12] Sandberg, I., Andrushchenko, Zh. N. and Pavlenko, V. P. 2005 Phys. Plasmas 12, 042311.

[13] Sharma, S., Das, A., Kaw, P. and Sen, A. 2007 Physica A 378, 211.

[14] Pokhotelov, O. A. and Onishchenko, O. G. 2011 Ann. Geophys. 29, 411.

[15] Kuvshinov, B. N. and Mikhailovskii, A. B. 1996 Plasma Phys. Rep. 22, 529.

[16] Onishchenko, O. G., Pokhotelov, O. A., Pavlenko, V. P., Shukla, P. K., Farid, T., Stenflo, L., Kamenets, F. F. and Bogdanov, A. V. 2001 Phys. Plasmas 8, 59.

[17] Onishchenko, O. G., Krasnoselskikh, V. V. and Pokhotelov, O. A. 2008 Phys. Plasmas 15, 022903. 